\title{
Modulation of Transient Receptor Potential C Channel Activity by Cholesterol
}

\author{
Rita Gutorov ${ }^{1}$, Maximilian Peters ${ }^{1}$, Ben Katz ${ }^{1}$, Tal Brandwine ${ }^{1}$, Nicolas A. Barbera2, \\ Irena Levitan ${ }^{2}$ and Baruch Minke ${ }^{1 *}$ \\ ${ }^{1}$ Institute for Medical Research Israel-Canada (IMRIC), Edmond and Lily Safra Center for Brain Sciences (ELSC), Faculty of \\ Medicine, The Hebrew University, Jerusalem, Israel, ${ }^{2}$ Division of Pulmonary, Critical Care, Sleep and Allergy, Department of \\ Medicine, University of Illinois at Chicago, Chicago, IL, United States
}

OPEN ACCESS

Edited by:

Moran Rubinstein,

Tel Aviv University, Israel

Reviewed by:

Bernard Attali,

Tel Aviv University, Israel

Ehud Ohana,

Ben-Gurion University of the

Negev, Israel

*Correspondence:

Baruch Minke

baruchm@ekmd.huji.ac.il

Specialty section:

This article was submitted to Pharmacology of lon Channels and Channelopathies, a section of the journal

Frontiers in Pharmacology

Received: 28 August 2019 Accepted: 15 November 2019 Published: 13 December 2019

Citation:

Gutorov R, Peters $M$, Katz B, Brandwine T, Barbera NA, Levitan I and Minke B (2019) Modulation of Transient Receptor Potential C Channel Activity by Cholesterol.

Front. Pharmacol. 10:1487. doi: 10.3389/fphar.2019.01487
Changes of cholesterol level in the plasma membrane of cells have been shown to modulate ion channel function. The proposed mechanisms underlying these modulations include association of cholesterol to a single binding site at a single channel conformation, association to a highly flexible cholesterol binding site adopting multiple poses, and perturbation of lipid rafts. These perturbations have been shown to induce reversible targeting of mammalian transient receptor potential C (TRPC) channels to the cholesterolrich membrane environment of lipid rafts. Thus, the observed inhibition of TRPC channels by methyl- $\beta$-cyclodextrin $(\mathrm{M} \beta \mathrm{CD})$, which induces cholesterol efflux from the plasma membrane, may result from disruption of lipid rafts. This perturbation was also shown to disrupt multimolecular signaling complexes containing TRPC channels. The Drosophila TRP and TRP-like (TRPL) channels belong to the TRPC channel subfamily. When the Drosophila TRPL channel was expressed in S2 or HEK293 cells and perfused with MBCD, the TRPL current was abolished in less than $100 \mathrm{~s}$, fitting well the fast kinetic phase of cholesterol sequestration experiments in cells. It was thus suggested that the fast kinetics of TRPL channel suppression by M $\beta C D$ arise from disruption of lipid rafts. Accordingly, lipid raft perturbation by cholesterol sequestration could give clues to the function of lipid environment in TRPC channel activity and its mechanism.

Keywords: TRP-like (TRPL) channel, lipid rafts, methyl- $\beta$-cyclodextrin, cholesterol recognition amino acid consensus sequence (CRAC), caveolae

\section{INTRODUCTION}

Cholesterol molecules are intercalated among the phospholipids of cell membrane forming an integral part of the plasma membrane, which is essential for the proper function of ion channels. Plasma membrane cholesterol includes domains known as lipid rafts (Pike, 2006). However, cholesterol is located in both rafts and non-raft fractions.

An efficient method to modulate the content of plasma membrane cholesterol is by methyl- $\beta$ cyclodextrin $(\mathrm{M} \beta \mathrm{CD})$, which is a cyclic oligosaccharide (Ohtani et al., 1989; Davis and Brewster, 2004). The $\beta$-cyclodextrins (seven glucose units) have high affinity for encapsulating cholesterol (Ohtani et al., 1989). M $\beta C D$ is quite specific for cholesterol, allowing enrichment or a relatively rapid sequestration of cholesterol from living cells. Cholesterol-saturated $\mathrm{M} \beta \mathrm{CD}$ is efficient as cholesterol donor. The degree of cholesterol enrichment is between $\sim 30 \%$ to $\sim$ threefold, according to the type 
of cell (Christian et al., 1997; Levitan et al., 2000). When cells are incubated with high concentration of "empty" M $\beta C D$ (5-10 $\mathrm{mM}$ ) for hours (> $2 \mathrm{~h}$ ), $80-90 \%$ of total cellular cholesterol can be sequestered (Kilsdonk et al., 1995; Levitan et al., 2000). The amount of cholesterol sequestration from different cell types is a highly variable parameter (Matthews et al., 1985; Kilsdonk et al., 1995; Christian et al., 1997; Niu et al., 2002). Cholesterol sequestration leads to dis-association of proteins from lipid rafts (Scheiffele et al., 1997; Kabouridis et al., 2000; Predescu et al., 2005) and to decrease in clustering of raft-associated molecules (Harder et al., 1998). Cholesterol depletion also disrupts caveolae structure; it does not result in the disappearance of caveolin but leads to a shift of caveolin from raft to non-raft fractions (Hissa et al., 2017) and to ruffling (Grimmer et al., 2002). It was shown that $\beta C D$ s sequestered cholesterol from both cholesterol-rich and cholesterol-poor membrane domains (Ottico et al., 2003; Gaus et al., 2005; Rouquette-Jazdanian et al., 2006; Tikku et al., 2007).

In this mini-review, we discuss physiological effects of modulating plasma membrane cholesterol. We focus on modulations of transient receptor potential C (TRPC) channels activity following application $\mathrm{M} \beta \mathrm{CD}$, with emphasis on fast modulations (in less than $100 \mathrm{~s}$ ).

\section{THE KINETICS OF CHOLESTEROL REMOVAL BY M $\beta C D$}

Cholesterol sequestration by $\beta C D$ from several cell types revealed bi-exponential kinetics, when monitored with radiolabeled $\left[\mathrm{H}^{3}\right]$ cholesterol: a fast ( $\tau 1 / 2$ of 19-23 s) and a slow ( $\tau$ 1/2 of 15-30 min) kinetics, suggesting the existence of two separate pools of cholesterol (Yancey et al., 1996). It was further suggested that the "fast" pool of cholesterol corresponds to the outer leaflet of the plasma membrane, while the identity of the slow pool was unclear (Yancey et al., 1996). Recently, imaging studies showed that cholesterol level in the inner leaflet of the plasma membrane was $\sim 12$-fold smaller than cholesterol concentration in the outer leaflet (Liu et al., 2017). Interestingly, two pools of $\mathrm{M} \beta \mathrm{CD}$ extracts were observed, with half-lives similar to those reported previously (Yancey et al., 1996; Haynes et al., 2000). However, it was also found that the slower cholesterol efflux (from the "slow" pool) was absent from energy-depleted cells (Hao et al., 2002).

The existence of a fast-modulated pool of cholesterol is important for the interpretation of studies showing fast responses of ion channels to modulations of cholesterol (see below). Unfortunately, most studies used prolonged incubations of cells and tissues with cyclodextrins, thus precluding the ability to observe fast kinetics of cholesterol modulations.

\section{MODULATION OF MAMMALIAN TRPC CHANNEL ACTIVITY BY CHOLESTEROL}

Cholesterol-ion channels interactions have been studied both computationally and experimentally. Earlier studies identified two types of cholesterol binding motifs: the cholesterol consensus motif (CCM) and the cholesterol recognition amino acid consensus sequence (CRAC), as well as the so-called CARC motif, in which the amino acid sequence appears in reverse. These motifs have been found in many ion channels, such as nicotinic acetylcholine receptor (nAChR), BK, P2X7, Kv1.3, as well as TRPV1 channels (Picazo-Juárez et al., 2011; Singh et al., 2012; Balajthy et al., 2017; Murrell-Lagnado, 2017). However, a recent analysis of the solved crystal structures of 24 cholesterol-protein complexes with 38 distinct cholesterol binding sites showed that fewer than $40 \%$ of these sites contained a CRAC or CARC motif, indicating that these motifs at best form only a subset of potential cholesterol binding sites (Rosenhouse-Dantsker, 2017). Furthermore, the relatively loose definition of the motif, (L/V)- $\mathrm{X}_{1-5^{-}}(\mathrm{Y})-\mathrm{X}_{1-5^{-}}(\mathrm{K} / \mathrm{R})$, where $\mathrm{X}$ can be one to five residues of any kind, has raised concerns about the predictive nature of the motif and the risk of identifying false positives (Epand, 2006; Jaipuria et al., 2018). Notably, while for many channels, there is little or no experimental confirmation for cholesterol interacting with these motifs, it was shown that a CRAC motif has a significant effect on cholesterol modulation of TRPV1 channel activity (Picazo-Juárez et al., 2011).

Because the above cholesterol binding motifs represent only a subset of potential cholesterol binding sites, there is a risk in using this approach. Consequently, more recent strategies for identifying binding sites have utilized computational approaches such as docking analyses and molecular dynamics simulations to identify potential sites in an unbiased manner. In particular, cholesterol binding sites were identified on $\mathrm{nAChR}$, as well as on inwardly rectifying potassium (Kir) channels, voltage-dependent anion channel (VDAC), and $\mathrm{GABA}_{\mathrm{A}}$ receptors (Brannigan et al., 2008; Rosenhouse-Dantsker et al., 2013; Hénin et al., 2014; Weiser et al., 2014). In these studies, docking analyses were first used to predict a set of candidate binding sites, which were then refined through short atomistic simulations and tested experimentally. Importantly, these binding sites did not contain the previously described cholesterol binding motifs. A limitation of this approach, however, is that atomistic simulations usually are not long enough to observe the dynamic behavior of the cholesterol molecule moving from the bilayer to the binding site. This limitation is addressed most recently, with the development of coarse-grained force fields such as the Martini force field, long $(\mu \mathrm{s})$ time-scale simulations of membrane proteins allowing for the dynamic binding and unbinding of cholesterol to target proteins, providing deeper insight into the mechanisms of cholesterol regulation (Cang et al., 2013; Genheden et al., 2017; Rouviere et al., 2017; Barbera et al., 2018). Using these approaches, most recent studies discovered that in contrast to most other ligands, cholesterol binding is highly flexible and cholesterol dynamically explores its binding site, adopting multiple poses in a "cloud," rather than occupying a single conformation (Gimpl, 2016; Genheden et al., 2017; Rouviere et al., 2017; Barbera et al., 2018). Recently electron cryo-microscopy structure of zebra fish TRPC4 $\left(\mathrm{TRPC}_{\mathrm{DR}}\right)$ channel in its unliganded closed state, at an overall resolution of $3.6 \AA$ was published (Vinayagam et al., 2018). The transmembrane S1-S6 helices structure revealed that in the pre-S1 elbow domain inside the membrane, a cavity is formed with helices S1 and S4, in which a density corresponding to a sterol is formed (Figure 1). Since the authors added cholesteryl hemisuccinate (CHS) during the purification of TRPC4 $4_{\mathrm{DR}}$, they 


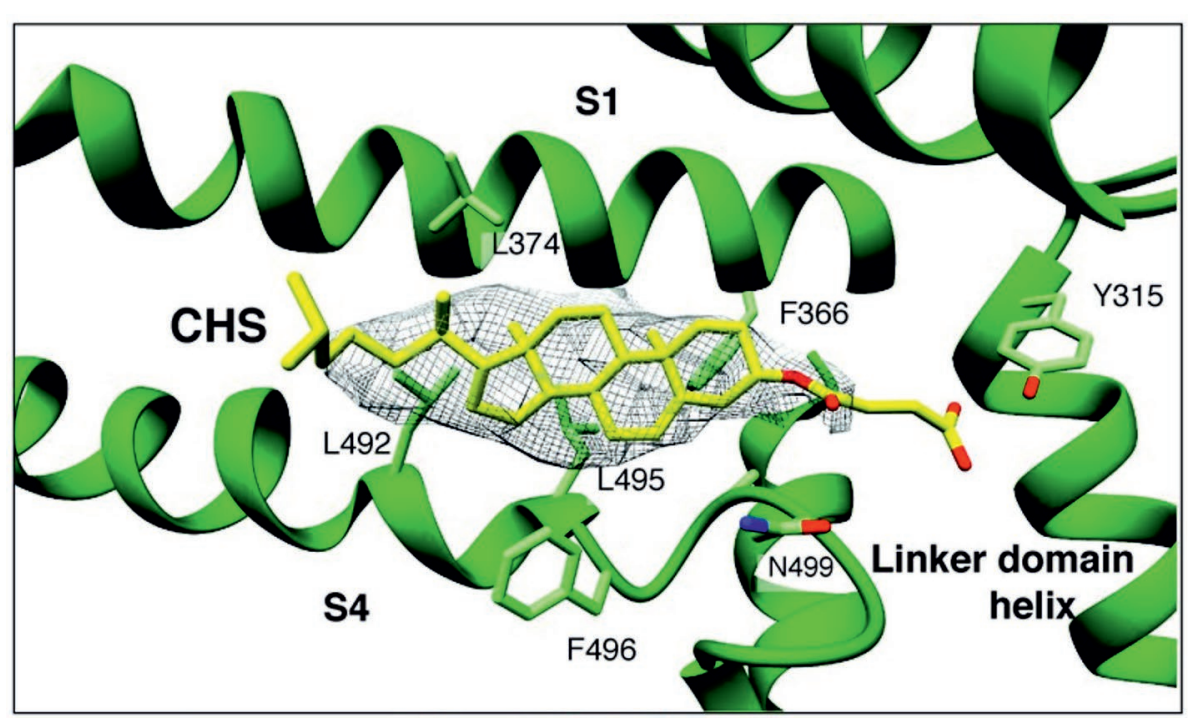

FIGURE 1 | A sterol binding pocket in the TRPC4 ${ }_{D R}$ structure. Electron cryo-microscopy structure of zebra fish TRPC4 (TRPC4 ${ }_{D R}$ ) channel in its unliganded closed state, at an overall resolution of $3.6 \AA$. The transmembrane S1-S6 helices structure revealed that in the pre-S1 elbow domain inside the membrane, a cavity is formed with helices S1 and S4, in which a density corresponding to a sterol is formed. (Reproduced from Vinayagam et al. (2018) with permission from eLife.)

fitted this molecule into the density. This density in the S1-S4 cavity is consistent with the above notion that sterol binding to channel proteins is flexible.

Cholesterol changes in the plasma membrane of cells have been shown to modulate ion channels function, and these modulations include perturbation of specific lipid environments. Experimental observations have suggested reversible targeting of mammalian TRPC channels to cholesterol-rich membrane environment of lipid rafts. This led to the suggestion that the observed inhibition of mammalian TRPC channels by M $\beta C D$-inducing cholesterol efflux may result in part from disruption of lipid rafts, including disruption of multimolecular signaling complexes (Svobodova and Groschner, 2016). Below we give examples for the role of lipid rafts in several types of TRPC channels.

\section{TRPC1}

The mammalian TRPC1 was shown to interact with caveolin-1 (Cav-1), which is a scaffolding protein that binds cholesterol (Lockwich et al., 2000). This interaction is mediated via both $\mathrm{N}$-terminal Cav-1 binding motif and C-terminal Cav-scaffolding consensus. The important role of caveolae in TRPC1 activation was supported by the finding that TRPC1 activity was dependent on Cav-1 (Murata et al., 2007), while TRPC1 was found mainly in caveolae (Lockwich et al., 2000). The experiments indicated that TRPC1 mainly resides in lipid rafts and exposed to the cholesterol-rich membrane of caveolae (Lockwich et al., 2000). Inhibition of TRPC1 currents by M $\beta C D$-induced cholesterol sequestration may result from both disruption of lipid raft architecture, including impaired local assembly of signaling molecules, and inhibition of a gating mechanism. The above two effects of cholesterol on TRPC1 function (i.e. disruption of lipid raft architecture and inhibition of a gating mechanism) was demonstrated for several cell types (Bergdahl et al., 2003; Brownlow and Sage, 2005; Kannan et al., 2007).

\section{TRPC3}

Cholesterol sensitivity of the TRPC3 channel was demonstrated by using acute administration of cholesterol-saturated $\mathrm{M} \beta \mathrm{CD}$ to modify membrane cholesterol content. Cholesterol application elevated conductance in TRPC3-expressing HEK293 cell culture. The membrane conductance derived from I-V curves was typical for phospholipase C (PLC)-mediated TRPC3 currents, showing fast rise time of several seconds. Thus, increased cholesterol concentrations induced a relatively fast TRPC3-mediated current in HEK293 cells (Graziani et al., 2006). Surface biotinylation experiments revealed a significant increase of TRPC3 level at the plasma membrane caused by cholesterol addition. This result suggests that TRPC3-mediated current and the ensuing $\mathrm{Ca}^{2+}$ influx that were induced by cholesterol elevation may resulted from a cholesterol-induced expression of TRPC3 in the surface membrane (Graziani et al., 2006).

Like TRPC1, the TRPC3 channels reside in caveolae. The significance of caveolae in mediating inositol 1,4,5 trisphosphate $\left(\mathrm{IP}_{3}\right)$-induced non-selective cation current $\left(\mathrm{I}_{\mathrm{Cat}}\right)$ activation and arterial smooth muscle constriction was studied in smooth muscles of cerebral arteries (Adebiyi et al., 2011). Immunoprecipitation and immunoFRET experiments revealed that Cav-1, TRPC3, and $\mathrm{IP}_{3}$ receptor1 $\left(\mathrm{IP}_{3} \mathrm{R} 1-1\right)$ formed a multimolecular signaling complex via Cav-1 scaffolding domain that was reversibly disrupted by $\mathrm{M} \beta \mathrm{CD}$ and by a peptide with Cav-1 scaffolding domain. These experiments revealed close association of the signaling proteins in smooth muscles of cerebral arteries. In other experiments, caveolae disassembly was obtained by: (1) M $\beta C D$, (2) Cav-1 knockdown using RNAi, or (3) 
application of Cav-1 scaffolding domain. Caveolae disassembly inhibited the $\mathrm{I}_{\text {Cat }}$ currents and vasoconstriction. The data thus indicated that the multimolecular signaling complex via Cav-1 scaffolding domain allowed signal-induced vasoconstriction (Adebiyi et al., 2011).

\section{TRPC6}

Podocytes are multipolar cells that cover the external surface of glomerular capillaries and form an essential component of the kidney ultrafiltration apparatus (Pavenstädt et al., 2003). Importantly, in order for podocytes to respond to distending forces, their $\mathrm{Ca}^{2+}$-dependent contractile elements must be coupled to a $\mathrm{Ca}^{2+}$ signaling pathway. The major source of regulated $\mathrm{Ca}^{2+}$ influx into podocytes is $\mathrm{Ca}^{2+}$-permeable TRPC6 channel, which like other mammalian TRPC channels are activated via PLC-mediated signaling cascade (Dryer and Reiser, 2010; Katz et al., 2017). Several mutations in the TRPC6 channel result in autosomal-dominant nephrotic syndromes (Reiser et al., 2005; Winn et al., 2005; Heeringa et al., 2009). Podocin is a cholesterol binding protein, which interacts, in a still unclear manner, with the TRPC6 channel (Reiser et al., 2005; Huber et al., 2006). The ability of podocin, which resides in lipid raft domains (Lei et al., 2014), to bind cholesterol may be central to TRPC6 gating (Huber et al., 2006). Inhibition of TRPC6 channels by application of $\mathrm{M} \beta \mathrm{CD}$ or by expression of dominant-negative Cav-1 isoform indicated that TRPC6 activation requires lipid rafts regions at the surface membrane (Lei et al., 2014), suggesting that podocin coordinates TRPC6 channel activity (Anderson et al., 2013).

\section{FAST INHIBITION OF THE DROSOPHILA TRP-LIKE CHANNEL ACTIVITY BY M $\beta C D$}

The Drosophila light-sensitive TRP and TRP-like (TRPL) channels are the first members of the TRPC subfamily that were discovered (review Katz et al., 2017). It is well established that the TRP/TRPL channels are the target of the rhodopsin-activatedphosphoinositide cascade, which leads to production of lipids that may function as second messenger in a variety of cells and tissues. Cyclodextrins, both $\alpha$-cyclodextrin and $\mathrm{M} \beta \mathrm{CD}$, are known to sequester phospholipids (Ohtani et al., 1989) that are involved directly or indirectly in gating of TRPC channels.

Light activation of PLC in Drosophila photoreceptors leads to the formation of diacylglycerol (DAG) and $\mathrm{IP}_{3}$, which are then recycled back to form phosphatidylinositol 4,5-bisphosphate [PtdIns $(4,5) \mathrm{P}_{2}$, designated the phosphoinositide (PI) cycle, Figure 2A). The mechanism by which the PI cycle activates the TRP/TRPL channels is not entirely clear (e.g. see Hardie, 2003). Nevertheless, the involvement of lipids in TRP/TRPL channel activation may account for the effects of M $B C D$ on the TRPL channel (see below).

To characterize the Drosophila light-sensitive channels, TRPL channels were expressed in tissue-cultured S2 (Hardie et al., 1997; Chyb et al., 1999; Parnas et al., 2009; Lev et al., 2012b; Peters et al., 2017) and HEK293 cells (Hambrecht et al., 2000; Lev et al., 2012b;
Peters et al., 2017). TRPL channels expressed in the Drosophila S2 cells showed basal activity that could beamplified by polyunsaturated fatty acid [PUFA, e.g. linoleic acid (LA), Lev and Minke, 2010; Lev et al., 2012a; Lev et al., 2012b]. The pronounced basal TRPL current obtained at positive voltage was virtually abolished by $\mathrm{M} \beta \mathrm{CD}$, in less than $100 \mathrm{~s}$ (Figures 2B-D). This quick effect of cholesterol sequestration is in marked contrast to the previously shown slow effect (of many minutes) of cholesterol sequestration (Singh et al., 2011). Inhibition of the TRPL current persisted long after removal of $\mathrm{M} \beta \mathrm{CD}$, excluding direct inhibition of TRPL by M $\beta C D$ (Figure 2C). In further experiments, cholesterol was first depleted by means of $\mathrm{M} \beta \mathrm{CD}$ in S2 cells expressing TRPL; then the excess of $M \beta C D$ was washed out, and the cells were perfused with the TRPL channel activator LA. This protocol initially resulted, as expected, in reduction of TRPL currents (see Figure 2), but surprisingly, LA could activate the TRPL channels independent of M $\beta C D$. These results suggest that in S2 cells, M $\beta C D$ does not affects the TRPL channels directly but affected G-protein coupled related signaling proteins upstream of TRPL in the cascade. Also, it is possible that cholesterol and LA share common mechanism of action, or that LA activation does not require cholesterol for its action.

To further examine at what stage of the transduction cascade $\mathrm{M} \beta \mathrm{CD}$ operates, we expressed the Pleckstrin Homology domain of PLC- $\delta$ attached to the green fluorescent protein (GFP) in tissue culture cells. The Pleckstrin Homology domain, which binds specifically PtdIns $(4,5) \mathrm{P}_{2}$ (and $\mathrm{IP}_{3}$ ), marks plasma membrane PtdIns $(4,5) \mathrm{P}_{2}$ in living cells (Balla and Varnai, 2002; Suh et al., 2006; Lev et al., 2012b). Since it is technically difficult to perform these experiments in S2 cells, HEK293 cells were used. In HEK293 cells expressing TRPL, no basal (constitutive) TRPL current was observed [(Figure 3A), unlike the situation in S2 cells (Figures 2B, C, Lev et al., 2012a)]. Under control conditions, the Pleckstrin Homology domain-GFP was associated with PtdIns $(4,5) \mathrm{P}_{2}$ of the surface membrane (Figures 3C-H). However, when $\operatorname{PtdIns}(4,5)$ $\mathrm{P}_{2}$ concentrations were reduced [e.g. by PLC-dependent hydrolysis of PtdIns $(4,5) \mathrm{P}_{2}$ ], the GFP-associated peptide diffused to the cell body (Figures $\mathbf{3 C}-\mathbf{H}$ ). PLC was activated by the expressed muscarinic receptor (hM1, which was activated by carbachol, CCH, Figures 3C, D), before (Figure 3C) and after application of $\mathrm{M} \beta \mathrm{CD}$ (Figure 3D). These experiments showed that $\mathrm{M} \beta C D$ had no effect on PtdIns $(4,5) \mathrm{P}_{2}$ hydrolysis by PLC, thus indicating that inhibition of TRPL-dependent current by cholesterol sequestration takes place after activation of PLC in the transduction cascade in HEK293 cells. Strikingly, application of LA, which is a highly potent activator of the TRPL channels, acting directly on the channels (Parnas et al., 2009) had virtually no effect (Figure 3B). Thus, in HEK293 cells, LA applied after response suppression by $\mathrm{M} \beta C D$ had virtually no effect on TRPL channel activity, suggesting that in HEK293 cells unlike S2 cells, cholesterol depletion suppress directly TRPL channel activation.

\section{CONCLUSION}

Cholesterol molecules are essential for the proper function of ion channels, including TRPC channels. Sequestration of cholesterol by $\mathrm{M} \beta \mathrm{CD}$ from the plasma membrane of cells expressing TRPC 


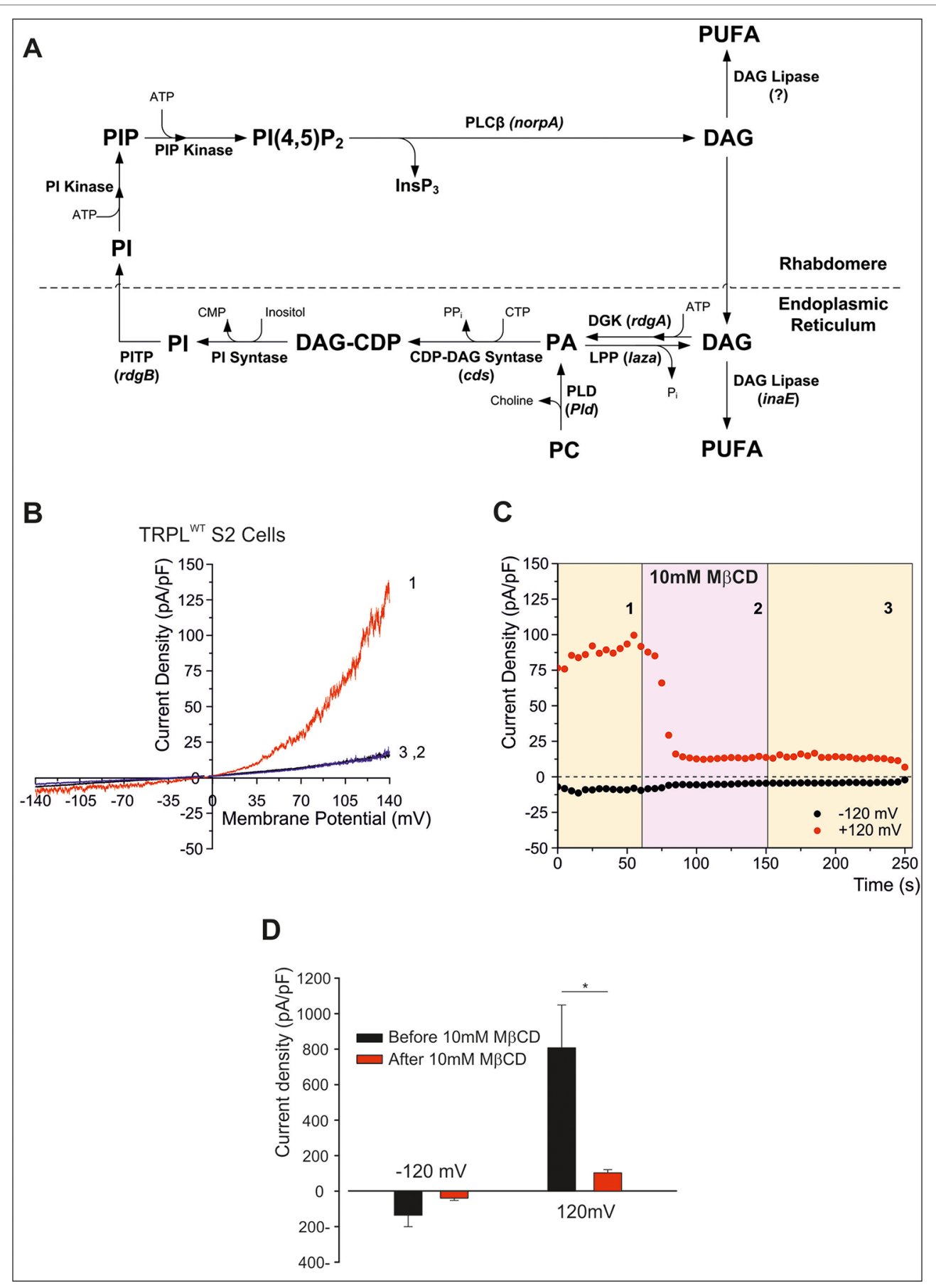

FIGURE 2 | (A) The phosphoinosite (PI) cycle. In the phototransduction cascade, light triggers the activation of phospholipase C $\beta$ (PLC $\beta$, encoded by norpA). This catalyzes hydrolysis of the membrane phospholipid $\mathrm{P}(4,5) \mathrm{P}_{2}\left(\mathrm{PIP}_{2}\right)$ into $\mathrm{IP}_{3}$ and diacylglycerol (DAG). DAG is transported by endocytosis to the endoplasmic reticulum and inactivated by phosphorylation converting it into phosphatidic acid (PA) via DAG kinase (DGK, encoded by rdgA) and to CDP-DAG via CDP-DAG synthase. Subsequently, CDP-DAG is converted into phosphatidyl inositol (PI), which is transferred back to the microvillar membrane, by the PI transfer protein (encoded by rdgB). PIP and PIP ${ }_{2}$ are produced at the microvillar membrane by PI kinase and PIP kinase, respectively. PA can also be converted back to DAG by lipid phosphate phosphohydrolase (Lpp, encoded by laza). PA is also produced from phosphatidyl choline (PC) by phospholipase D (PLD). DAG is also converted in two enzymatic stages, one of them is by DAG lipase (encoded by inaE), into polyunsaturated fatty acids (PUFAs). (B-D) MBCD blocks constitutive TRPL channels activity. (B) Current-voltage relationships (I-V curves) measuring TRPL-dependent currents. $1-V$ curves obtained in response to voltage ramp (of $1 \mathrm{~s}$ duration) from S2 cells expressing TRPL and showing basal channel activity with strong outward rectification, typical for TRPL-dependent current (1). The TRPL channel activity was highly reduced after perfusion with $10 \mathrm{mM}$ methyl- $\beta$-cyclodextrin (MBCD) (2) and the effect was irreversible, even after washout of $M \beta C D(3)(n>10)$. (C) Time course of the M $B C D$ effects on TRPL currents in $S 2$ cells. Current densities are shown as a function of time. Series of $I-V$ curves were derived from repeatedly applied voltage ramps every $5 \mathrm{~s}$, and currents were measured at $\pm 120 \mathrm{mV}$ holding potentials as a function of time under the various experimental conditions as indicated. The numbers correspond to the numbers on the I-V curves in (B). (D) Statistics of the cholesterol depletion experiments in S2 cells. (A) Cholesterol depletion by MBCD had a significant effect on the positive TRPL currents at $120 \mathrm{mV}\left(n=5\right.$, values are average \pm SEM, paired Student $t$-test, $\left.{ }^{*} p \leq 0.05\right)$. Reproduced from Katz and Minke, 2009 with permission from Frontiers. (B-D) Reproduced from Peters et al., 2017 with permission from Elsevier, license number 4676401165468.) 
channels suppressed channel activity. It is still not entirely clear what is the mechanism underlying suppression of TRPC channels activity by $\mathrm{M} \beta \mathrm{CD}$. Possible mechanisms include association of cholesterol to a highly flexible cholesterol binding site or perturbation of specific lipid environments. Mammalian TRPC1 and TRPC6 channels require for their activity binding to scaffold proteins located in cholesterol-rich lipid rafts. For these channels, inhibition resulting from cholesterol sequestration by $\mathrm{M} \beta \mathrm{CD}$ possibly resulted from disruption of lipid rafts at the plasma membrane, rather than direct inhibition of a gating mechanism. Nevertheless, channel-cholesterol interactions similar to those reported for Kir channels cannot be excluded. Future availability of atomic structure of TRPC channel subfamilies nanodisks can be very useful to determine the possibility that in contrast
A

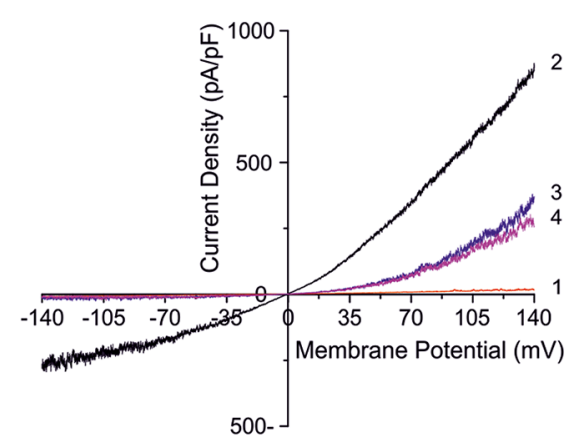

C

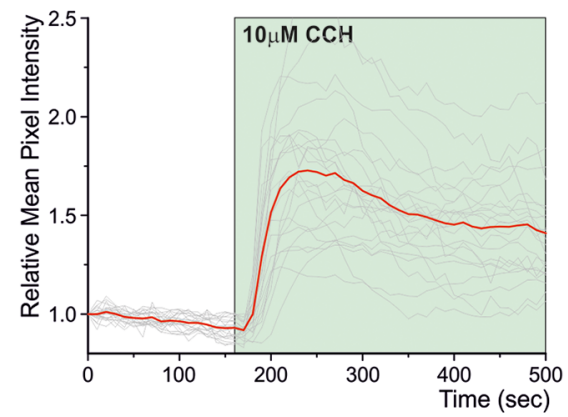

E

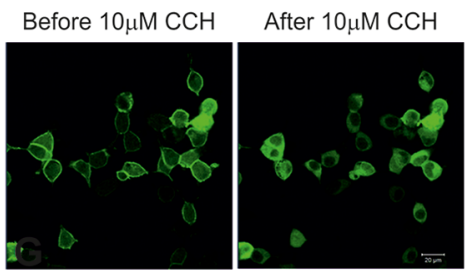

G
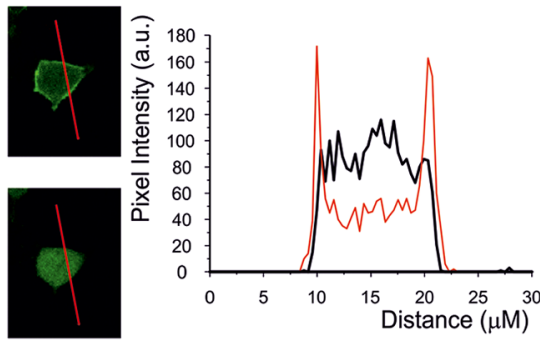

B

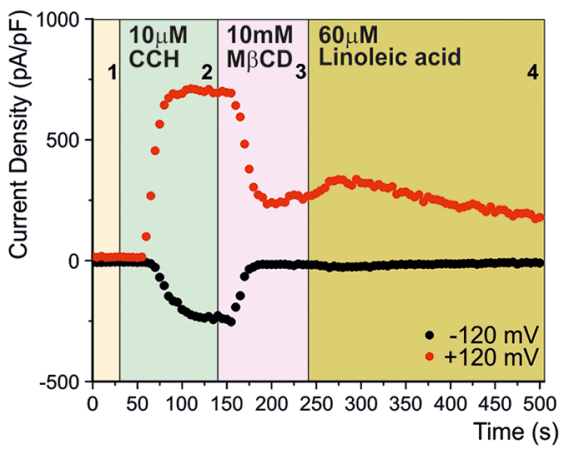

D

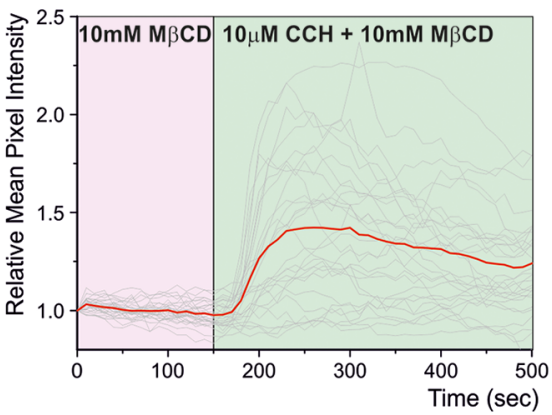

F

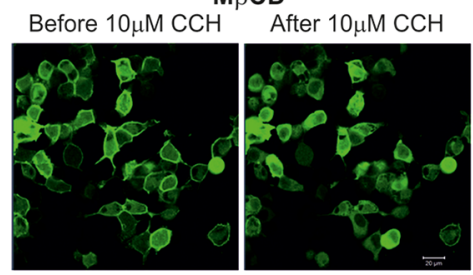

$\mathrm{H}$

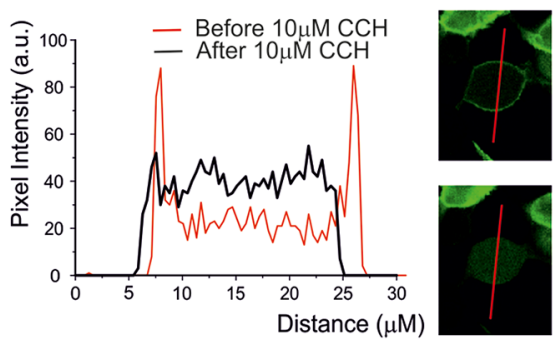


FIGURE 3 | (A-B) Cholesterol depletion suppressed receptor-activated TRPL-dependent current. (A) TRP-like-green fluorescent protein (TRPL-GFP) did not show any spontaneous activity in HEK293 cells, but it could be readily activated via PLC and blocked by M 3 CD: current-voltage relationships measured from HEK293 cells expressing TRPL-GFP, showing no basal channel activity (1). However, coexpression of the hM1 muscarinic receptor and application of carbachol (CCH) activated the expressed TRPL-GFP channels via endogenous PLC-mediated cascade (2) and the TRPL-dependent current in the presence of CCH was suppressed by application of $M \beta C D$ (3), while subsequent application of LA, a strong activator of TRPL channels, did not activate the channels after the application of M $\beta C D$ (4). (B) Time course of the receptor-activated TRPL-dependent current and the effect of cholesterol depletion on the receptor-activated TRPL currents in HEK293 cells. Current densities are shown as a function of time. Series of $\mathrm{i}-\mathrm{V}$ curves were derived from repeatedly applied voltage ramps every $5 \mathrm{~s}$, and currents were measured at $\pm 120 \mathrm{mV}$ holding potentials as a function of time under the various experimental conditions as indicated (C-H) Cholesterol depletion did not affect receptor-induced PLC activity. No effects of cholesterol depletion on PLC activity as monitored by translocation of the PIP ${ }_{2}$ sensor PH-GFP: representative series of multiphoton images of HEK293 cells coexpressing eGFP-tagged PH domain and hM1 receptor. Application of CCH to the bathing solution, in a concentration that activated the TRPL channels $(10 \mu \mathrm{M} \mathrm{CCH})$, induced similar translocation of the eGFP-tagged PH domain to the cell body, with and without $\mathrm{M} \beta \mathrm{CD}$, indicating the PLC-mediated hydrolysis of $\mathrm{PIP}_{2}$ is not affected by M $\beta C D$. (C) The time course of fluorescence changes measured in the cytosol before application of $\mathrm{M} \beta \mathrm{CD}$ : graph plotting the relative mean pixels' intensity (red curve) as a function of time measured from multiphoton images of HEK293 cells expressing PH-GFP and hM1 receptors. Before PLC stimulation by $\mathrm{CCH}$ application (white background), the GFP-PH is associated with the plasma membrane where most PIP ${ }_{2}$ is located and the cell body fluorescence is low (for quantification, see $\mathbf{G}, \mathbf{H}$ ). Once PLC is activated and PIP ${ }_{2}$ is hydrolyzed (green background), the PH-GFP translocates to the cytosol and there is a marked increase in fluorescence intensity at the cytosol. The individual single-cell measurements are shown by noisy dim gray traces. (D) The time course of fluorescence changes measured in the cytosol after application of M $\beta C D$ : similar graph as in (C), but measured following application of M $\beta C D$. (E) Multiphoton images of HEK293 cells expressing PH-GFP and hM1 receptor without application of M $\beta C D$ : Left: GFP fluorescence of cells before application of $\mathrm{CCH}$, little PH-GFP translocation was observed. Right: GFP fluorescence of cells after perfusion with the M1 agonist CCH. Translocation of PH-GFP is observed. M $\beta C D$ was not applied ( $\mathrm{n}>50)$. (F) Multiphoton images of HEK293 cells expressing PH-GFP and hM1 receptor after application of M $\beta$ CD: Similar images of HEK293 cells expressing TRPL PH-GFP and hM1 receptor before (left) and after application of CCH (right). M $\beta C D$ was applied, but it did not affect PHGFP translocation $(n>50)$. (G, H) Graphs plotting the PH-GFP fluorescence intensity as a function of cell position: fluorescence intensity of images showing cross sections of two representative cells along the red line, before application of $\mathrm{CCH}$ (red curve), and after application of $\mathrm{CCH}$ (black curve) in the absence of $\mathrm{M} \beta \mathrm{CD}$ (G) and after application of M $\beta C D(\mathbf{H})$. (Reproduced from Peters et al., 2017 with permission from Elsevier, license number 4676401165468.)

to most other ligands, cholesterol binding to TRPC channels is highly flexible and cholesterol dynamically explores its binding site, adopting multiple poses in a "cloud," rather than occupying a single conformation and in this way affects channel gating. For the Drosophila TRPL channel, where the gating mechanism of the channel is still unknown, elucidating the mechanism of cholesterol action may help solve the long-standing enigma of channel gating.

\section{AUTHOR CONTRIBUTIONS}

RG presented the lecture in the Israel Ion Channel and Transporters Meeting 2019, conducted a wide literature search, drafted the manuscript, and participated in the writing. MP

\section{REFERENCES}

Adebiyi, A., Narayanan, D., and Jaggar, J. H. (2011). Caveolin-1 assembles type 1 inositol 1,4,5-trisphosphate receptors and canonical transient receptor potential 3 channels into a functional signaling complex in arterial smooth muscle cells. J. Biol. Chem. 286, 4341-4348. doi: 10.1074/ jbc.M110.179747

Anderson, M., Kim, E. Y., Hagmann, H., Benzing, T., and Dryer, S. E. (2013). Opposing effects of podocin on the gating of podocyte TRPC6 channels evoked by membrane stretch or diacylglycerol. Am. J. Physiol. Cell Physiol. 305, C276-C289. doi: 10.1152/ajpcell.000952013

Balajthy, A., Hajdu, P., Panyi, G., and Varga, Z. (2017). Sterol Regulation of VoltageGated K. Curr. Top. Membr 80, 255-292. doi: 10.1016/bs.ctm.2017.05.006

Balla, T., and Varnai, P. (2002). Visualizing cellular phosphoinositide pools with GFP-fused protein-modules. SciSTKE L3. 1-17. doi: 10.1126/ scisignal.1252002pl3

Barbera, N., Ayee, M. A. A., Akpa, B. S., and Levitan, I. (2018). Molecular dynamics simulations of kir2.2 interactions with an ensemble of cholesterol molecules. Biophys. J. 115, 1264-1280. doi: 10.1016/j.bpj.2018.07.041

Bergdahl, A., Gomez, M. F., Dreja, K., Xu, S. Z., Adner, M., Beech, D. J., et al. (2003). Cholesterol depletion impairs vascular reactivity to endothelin-1 by performed the experimental part of the review and conducted part of the literature search. TB conducted part of the literature search. BK participated in the writing and drafting of the manuscript. NB wrote the section on the Computational Approach and inserted the relevant literature. IL covered the Computational Approach, searched for the relevant literature, and participated in the writing. BM initiated the review, drafted the manuscript, and wrote the paper.

\section{ACKNOWLEDGMENTS}

This study was supported by the United States-Israel Binational Science Foundation grant no. 2015014 (BM and IL).

reducing store-operated Ca2+ entry dependent on TRPC1. Circ. Res. 93, 839847. doi: 10.1161/01.RES.0000100367.45446.A3

Brannigan, G., Hénin, J., Law, R., Eckenhoff, R., and Klein, M. L. (2008). Embedded cholesterol in the nicotinic acetylcholine receptor. Proc. Natl. Acad. Sci. U.S.A. 105, 14418-14423. doi: 10.1073/pnas.0803029105

Brownlow, S. L., and Sage, S. O. (2005). Transient receptor potential protein subunit assembly and membrane distribution in human platelets. Thromb. Haemost. 94, 839-845. doi: 10.1160/TH05-06-0391

Cang, X., Du, Y., Mao, Y., Wang, Y., Yang, H., and Jiang, H. (2013). Mapping the functional binding sites of cholesterol in $\beta 2$-adrenergic receptor by longtime molecular dynamics simulations. J. Phys. Chem. B 117, 1085-1094. doi: $10.1021 /$ jp3118192

Christian, A. E., Haynes, M. P., Phillips, M. C., and Rothblat, G. H. (1997). Use of cyclodextrins for manipulating cellular cholesterol content. J. Lipid Res. 38, 2264-2272.

Chyb, S., Raghu, P., and Hardie, R. C. (1999). Polyunsaturated fatty acids activate the Drosophila light-sensitive channels TRP and TRPL. Nature 397, 255-259. doi: $10.1038 / 16703$

Davis, M. E., and Brewster, M. E. (2004). Cyclodextrin-based pharmaceutics: past, present and future. Nat. Rev. Drug Discovery 3, 1023-1035. doi: 10.1038/ nrd1576 
Dryer, S. E., and Reiser, J. (2010). TRPC6 channels and their binding partners in podocytes: role in glomerular filtration and pathophysiology. Am. J. Physiol. Renal Physiol. 299, F689-F701. doi: 10.1152/ajprenal.002982010

Epand, R. M. (2006). Cholesterol and the interaction of proteins with membrane domains. Prog. Lipid Res. 45, 279-294. doi: 10.1016/j.plipres.2006.02.001

Gaus, K., Rodriguez, M., Ruberu, K. R., Gelissen, I., Sloane, T. M., Kritharides, L., et al. (2005). Domain-specific lipid distribution in macrophage plasma membranes. J. Lipid Res. 46, 1526-1538. doi: 10.1194/jlr.M500103-JLR200

Genheden, S., Essex, J. W., and Lee, A. G. (2017). G protein coupled receptor interactions with cholesterol deep in the membrane. Biochim. Biophys. Acta Biomembr. 1859, 268-281. doi: 10.1016/j.bbamem.2016.12.001

Gimpl, G. (2016). Interaction of $\mathrm{G}$ protein coupled receptors and cholesterol. Chem. Phys. Lipids 199, 61-73. doi: 10.1016/j.chemphyslip.2016.04.006

Graziani, A., Rosker, C., Kohlwein, S. D., Zhu, M. X., Romanin, C., Sattler, W., et al. (2006). Cellular cholesterol controls TRPC3 function: evidence from a novel dominant-negative knockdown strategy. Biochem. J. 396, 147-155. doi: 10.1042/BJ20051246

Grimmer, S., van Deurs, B., and Sandvig, K. (2002). Membrane ruffling and macropinocytosis in A431 cells require cholesterol. J. Cell Sci. 115, 2953-2962.

Hénin, J., Salari, R., Murlidaran, S., and Brannigan, G. (2014). A predicted binding site for cholesterol on the GABAA receptor. Biophys. J. 106, 1938-1949. doi: 10.1016/j.bpj.2014.03.024

Hambrecht, J., Zimmer, S., Flockerzi, V., and Cavalie, A. (2000). Single-channel currents through transient-receptor-potential-like (TRPL) channels. Pflugers Arch. 440, 418-426. doi: 10.1007/s004240000301

Hao, M., Lin, S. X., Karylowski, O. J., Wüstner, D., McGraw, T. E., and Maxfield, F. R. (2002). Vesicular and non-vesicular sterol transport in living cells. The endocytic recycling compartment is a major sterol storage organelle. J. Biol. Chem. 277, 609-617. doi: 10.1074/jbc.M108861200

Harder, T., Scheiffele, P., Verkade, P., and Simons, K. (1998). Lipid domain structure of the plasma membrane revealed by patching of membrane components. $J$. Cell Biol. 141, 929-942. doi: 10.1083/jcb.141.4.929

Hardie, R. C., Reuss, H., Lansdell, S. J., and Millar, N. S. (1997). Functional equivalence of native light-sensitive channels in the Drosophila trp 301 mutant and TRPL cation channels expressed in a stably transfected Drosophila cell line. Cell Calcium 21, 431-440. doi: 10.1016/S0143-4160(97)90054-3

Hardie, R. C. (2003). Regulation of trp channels via lipid second messengers. AnnuRevPhysiol 65, 735-759. doi: 10.1146/annurev.physiol.65.092101.142505

Haynes, M. P., Phillips, M. C., and Rothblat, G. H. (2000). Efflux of cholesterol from different cellular pools. Biochemistry 39, 4508-4517. doi: 10.1021/bi992125q

Heeringa, S. F., Möller, C. C., Du, J., Yue, L., Hinkes, B., Chernin, G., et al. (2009). A novel TRPC6 mutation that causes childhood FSGS. PloS One 4, e7771. doi: 10.1371/journal.pone.0007771

Hissa, B., Oakes, P. W., Pontes, B., Ramírez-San Juan, G., and Gardel, M. L. (2017). Cholesterol depletion impairs contractile machinery in neonatal rat cardiomyocytes. Sci. Rep. 7, 43764. doi: 10.1038/srep43764

Huber, T. B., Schermer, B., Müller, R. U., Höhne, M., Bartram, M., Calixto, A., et al. (2006). Podocin and MEC-2 bind cholesterol to regulate the activity of associated ion channels. Proc. Natl. Acad. Sci. U.S.A. 103, 17079-17086. doi: 10.1073/pnas.0607465103

Jaipuria, G., Giller, K., Leonov, A., Becker, S., and Zweckstetter, M. (2018). Insights into Cholesterol/Membrane Protein Interactions Using Paramagnetic SolidState NMR. Chemistry 24, 17606-17611. doi: 10.1002/chem.201804550

Kabouridis, P. S., Janzen, J., Magee, A. L., and Ley, S. C. (2000). Cholesterol depletion disrupts lipid rafts and modulates the activity of multiple signaling pathways in T lymphocytes. Eur. J. Immunol. 30, 954-963. doi: 10.1002/1521-4141(200003)30:3<954::AID-IMMU954>3.0.CO;2-Y

Kannan, K. B., Barlos, D., and Hauser, C. J. (2007). Free cholesterol alters lipid raft structure and function regulating neutrophil $\mathrm{Ca} 2+$ entry and respiratory burst: correlations with calcium channel raft trafficking. J. Immunol. 178, 5253-5261. doi: 10.4049/jimmunol.178.85253

Katz, B., and Minke, B. (2009). Drosophila photoreceptors and signaling mechanisms. (FL: Boca Raton) Front. Cell Neurosci. 3, 2. doi: 10.3389/ neuro.03.0022009

Katz, B., Payne, R., and Minke, B. (2017). TRP Channels in Vision (Boca Raton (FL): CRC Press, Taylor \& Francis Group). doi: 10.4324/9781315152837-3

Kilsdonk, E. P., Yancey, P. G., Stoudt, G. W., Bangerter, F. W., Johnson, W. J., Phillips, M. C., et al. (1995). Cellular cholesterol efflux mediated by cyclodextrins. J. Biol. Chem. 270, 17250-17256. doi: 10.1074/jbc.270.29.17250
Lei, L., Lu, S., Wang, Y., Kim, T., and Mehta, D. (2014). The role of mechanical tension on lipid raft dependent PDGF-induced TRPC6 activation. Biomaterials 35, 2868-2877. doi: 10.1016/j.biomaterials.2013.12.030

Lev, S., and Minke, B. (2010). Constitutive activity of TRP channels methods for measuring the activity and its outcome. Methods Enzymol. 484, 591-612. doi: 10.1016/B978-0-12-381298-8.00029-0

Lev, S., Katz, B., and Minke, B. (2012a). The activity of the TRP-like channel depends on its expression system. Channels (Austin) 6, 86-93. doi: 10.4161/ chan.19946

Lev, S., Katz, B., Tzarfaty, V., and Minke, B. (2012b). Signal-dependent hydrolysis of phosphatidylinositol 4,5-bisphosphate without activation of phospholipase C: implications on gating of Drosophila TRPL (transient receptor potentiallike) channel. J. Biol. Chem. 287, 1436-1447. doi: 10.1074/jbc.M111.266585

Levitan, I., Christian, A. E., Tulenko, T. N., and Rothblat, G. H. (2000). Membrane cholesterol content modulates activation of volume-regulated anion current in bovine endothelial cells. J. Gen. Physiol. 115, 405-416. doi: 10.1085/jgp.115.4.405

Liu, S. L., Sheng, R., Jung, J. H., Wang, L., Stec, E., O'Connor, M. J., et al. (2017). Orthogonal lipid sensors identify transbilayer asymmetry of plasma membrane cholesterol. Nat. Chem. Biol. 13, 268-274. doi: 10.1038/nchembio2268

Lockwich, T. P., Liu, X., Singh, B. B., Jadlowiec, J., Weiland, S., and Ambudkar, I. S. (2000). Assembly of Trp1 in a signaling complex associated with caveolinscaffolding lipid raft domains. J. Biol. Chem. 275, 11934-11942. doi: 10.1074/ jbc.275.16.11934

Matthews, D. A., Bolin, J. T., Burridge, J. M., Filman, D. J., Volz, K. W., Kaufman, B. T., et al. (1985). Refined crystal structures of Escherichia coli and chicken liver dihydrofolate reductase containing bound trimethoprim. J. Biol. Chem. 260, 381-391.

Murata, T., Lin, M. I., Stan, R. V., Bauer, P. M., Yu, J., and Sessa, W. C. (2007). Genetic evidence supporting caveolae microdomain regulation of calcium entry in endothelial cells. J. Biol. Chem. 282, 16631-16643. doi: 10.1074/jbc. M607948200

Murrell-Lagnado, R. D. (2017). Regulation of P2X purinergic receptor signaling by cholesterol. Curr. Top. Membr. 80, 211-232. doi: 10.1016/bs.ctm.2017.05.004

Niu, S. L., Mitchell, D. C., and Litman, B. J. (2002). Manipulation of cholesterol levels in rod disk membranes by methyl-beta-cyclodextrin: effects on receptor activation. J. Biol. Chem. 277, 20139-20145. doi: 10.1074/jbc.M200594200

Ohtani, Y., Irie, T., Uekama, K., Fukunaga, K., and Pitha, J. (1989). Differential effects of alpha-, beta- and gamma-cyclodextrins on human erythrocytes. Eur. J. Biochem. 186, 17-22. doi: 10.1111/j.1432-1033.1989.tb15171.x

Ottico, E., Prinetti, A., Prioni, S., Giannotta, C., Basso, L., Chigorno, V., et al. (2003). Dynamics of membrane lipid domains in neuronal cells differentiated in culture. J. Lipid Res. 44, 2142-2151. doi: 10.1194/jlr.M300247-JLR200

Parnas, M., Peters, M., and Minke, B. (2009). Linoleic acid inhibits TRP channels with intrinsic voltage sensitivity: implications on the mechanism of linoleic acid action. Channels (Austin) 3, 164-166. doi: 10.4161/chan.3.38873

Pavenstädt, H., Kriz, W., and Kretzler, M. (2003). Cell biology of the glomerular podocyte. Physiol. Rev. 83, 253-307. doi: 10.1152/physrev.000202002

Peters, M., Katz, B., Lev, S., Zaguri, R., Gutorov, R., and Minke, B. (2017). Depletion of Membrane Cholesterol Suppresses Drosophila Transient Receptor PotentialLike (TRPL) Channel Activity. Curr. Top. Membr 80, 233-254. doi: 10.1016/ bs.ctm.2017.05.005

Picazo-Juárez, G., Romero-Suárez, S., Nieto-Posadas, A., Llorente, I., JaraOseguera, A., Briggs, M., et al. (2011). Identification of a binding motif in the S5 helix that confers cholesterol sensitivity to the TRPV1 ion channel. J. Biol. Chem. 286, 24966-24976. doi: 10.1074/jbc.M111.237537

Pike, L. J. (2006). Rafts defined: a report on the Keystone Symposium on Lipid Rafts and Cell Function. J. Lipid Res. 47, 1597-1598. doi: 10.1194/jlr.E600002-JLR200

Predescu, S. A., Predescu, D. N., Shimizu, K., Klein, I. K., and Malik, A. B. (2005). Cholesterol-dependent syntaxin-4 and SNAP-23 clustering regulates caveolar fusion with the endothelial plasma membrane. J. Biol. Chem. 280, 3713037138. doi: 10.1074/jbc.M505659200

Reiser, J., Polu, K. R., Möller, C. C., Kenlan, P., Altintas, M. M., Wei, C., et al. (2005). TRPC6 is a glomerular slit diaphragm-associated channel required for normal renal function. Nat. Genet. 37, 739-744. doi: 10.1038/ng1592

Rosenhouse-Dantsker, A., Noskov, S., Durdagi, S., Logothetis, D. E., and Levitan, I. (2013). Identification of novel cholesterol-binding regions in Kir2 channels. J. Biol. Chem. 288, 31154-31164. doi: 10.1074/jbc.M113.496117

Rosenhouse-Dantsker, A. (2017). Insights into the molecular requirements for cholesterol binding to ion channels. Curr. Top. Membr 80, 187-208. doi: 10.1016/bs.ctm.2017.05.003 
Rouquette-Jazdanian, A. K., Pelassy, C., Breittmayer, J. P., and Aussel, C. (2006). Revaluation of the role of cholesterol in stabilizing rafts implicated in $\mathrm{T}$ cell receptor signaling. Cell Signal 18, 105-122. doi: 10.1016/j.cellsig.2005.03.024

Rouviere, E., Arnarez, C., Yang, L., and Lyman, E. (2017). Identification of two new cholesterol interaction sites on the A. Biophys. J. 113, 2415-2424. doi: 10.1016/j. bpj.2017.09.027

Scheiffele, P., Roth, M. G., and Simons, K. (1997). Interaction of influenza virus haemagglutinin with sphingolipid-cholesterol membrane domains via its transmembrane domain. EMBO J. 16, 5501-5508. doi: 10.1093/ emboj/16.185501

Singh, D. K., Shentu, T. P., Enkvetchakul, D., and Levitan, I. (2011). Cholesterol regulates prokaryotic Kir channel by direct binding to channel protein. Biochim. Biophys. Acta 1808, 2527-2533. doi: 10.1016/j. bbamem.2011.07.006

Singh, A. K., McMillan, J., Bukiya, A. N., Burton, B., Parrill, A. L., and Dopico, A. M. (2012). Multiple cholesterol recognition/interaction amino acid consensus (CRAC) motifs in cytosolic C tail of Slo1 subunit determine cholesterol sensitivity of $\mathrm{Ca} 2+-$ and voltage-gated $\mathrm{K}+(\mathrm{BK})$ channels. J. Biol. Chem. 287, 20509-20521. doi: 10.1074/jbc.M112.356261

Suh, B. C., Inoue, T., Meyer, T., and Hille, B. (2006). Rapid chemically induced changes of PtdIns(4,5)P2 gate KCNQ ion channels. Science 314, 1454-1457. doi: 10.1126/science. 1131163

Svobodova, B., and Groschner, K. (2016). Reprint of "Mechanisms of lipid regulation and lipid gating in TRPC channels". Cell Calcium 60, 133-141. doi: 10.1016/j.ceca.2016.06.010

Tikku, S., Epshtein, Y., Collins, H., Travis, A. J., Rothblat, G. H., and Levitan, I. (2007). Relationship between Kir2.1/Kir2.3 activity and their distributions between cholesterol-rich and cholesterol-poor membrane domains. Am. J. Physiol. Cell Physiol. 293, C440-C450. doi: 10.1152/ajpcell.004922006

Vinayagam, D., Mager, T., Apelbaum, A., Bothe, A., Merino, F., Hofnagel, O., et al. (2018). Electron cryo-microscopy structure of the canonical TRPC4 ion channel. Elife 7, 1-23. doi: 10.7554/eLife.36615

Weiser, B. P., Salari, R., Eckenhoff, R. G., and Brannigan, G. (2014). Computational investigation of cholesterol binding sites on mitochondrial VDAC. J. Phys. Chem. B 118, 9852-9860. doi: 10.1021/jp504516a

Winn, M. P., Conlon, P. J., Lynn, K. L., Farrington, M. K., Creazzo, T., Hawkins, A. F., et al. (2005). A mutation in the TRPC6 cation channel causes familial focal segmental glomerulosclerosis. Science 308, 1801-1804. doi: 10.1126/science.1106215

Yancey, P. G., Rodrigueza, W. V., Kilsdonk, E. P., Stoudt, G. W., Johnson, W. J., Phillips, M. C., et al. (1996). Cellular cholesterol efflux mediated by cyclodextrins. Demonstration Of kinetic pools and mechanism of efflux. J. Biol. Chem. 271, 16026-16034. doi: 10.1074/jbc.271.27.16026

Conflict of Interest: The authors declare that the research was conducted in the absence of any commercial or financial relationships that could be construed as a potential conflict of interest.

Copyright (C) 2019 Gutorov, Peters, Katz, Brandwine, Barbera, Levitan and Minke. This is an open-access article distributed under the terms of the Creative Commons Attribution License (CC BY). The use, distribution or reproduction in other forums is permitted, provided the original author(s) and the copyright owner(s) are credited and that the original publication in this journal is cited, in accordance with accepted academic practice. No use, distribution or reproduction is permitted which does not comply with these terms. 